\title{
Recency of HIV infection, antiretroviral therapy use and viral loads among symptomatic sexually transmitted infection service attendees in South Africa
}

\author{
T Kufa, ${ }^{1,2}$ MB ChB, PhD (D; F Radebe, ${ }^{1}$ BSc, MSc (Virol); E Cutler, ${ }^{1}$ BSc, MSc (Virol); M Goosen, ${ }^{1}$ BSc, BSc Hons (Med Virol); \\ L Wiesner, ${ }^{3} \mathrm{PhD}$; D Greyling, ${ }^{1}$ BSc Hons (Biochem); V Maseko, ${ }^{1}$ BTech; R Kularatne, ${ }^{1,4} \mathrm{MB}$ ChB, MMed (Micro); \\ A Puren, ${ }^{1,5} \mathrm{MB} \mathrm{BCh}, \mathrm{PhD}$ \\ ${ }^{1}$ Centre for HIV and STIs, National Institute for Communicable Diseases, Johannesburg, South Africa \\ ${ }^{2}$ School of Public Health, Faculty of Health Sciences, University of the Witwatersrand, Johannesburg, South Africa \\ ${ }^{3}$ Division of Clinical Pharmacology, Department of Medicine, Faculty of Health Sciences, University of Cape Town, South Africa \\ ${ }^{4}$ Department of Clinical Microbiology, School of Pathology, Faculty of Health Sciences, University of the Witwatersrand, Johannesburg, South Africa \\ ${ }^{5}$ Division of Virology, School of Pathology, Faculty of Health Sciences, University of the Witwatersrand, Johannesburg, South Africa
}

Corresponding author: T Kufa (tendesayikc@nicd.ac.za, tendesayik@yahoo.com)

\begin{abstract}
Background. Better integration of HIV and sexually transmitted infection (STI) prevention and treatment services is needed to accelerate progress towards the goal of zero new HIV infections.

Objectives. To describe HIV positivity, antiretroviral therapy (ART) use, viral suppression and recency of HIV infection among symptomatic STI service attendees at two primary care clinics in South Africa.

Methods. In a cross-sectional study, male and female STI service attendees presenting with symptoms consistent with STI syndromes were enrolled following informed consent. An interviewer-administered questionnaire was completed and appropriate genital and blood specimens were collected for STI testing and HIV biomarker measurements including recency of infection and antiretroviral (ARV) drug levels. Descriptive statistics were used to describe enrolled attendees, and to determine the proportion of attendees who were HIV-positive, recently infected, taking ART and virally suppressed. HIV-positive attendees with detectable ARVs were considered to be on ART, while those with viral loads (VLs) $\leq 200$ copies/mL were considered virally suppressed.

Results. Of 451 symptomatic attendees whose data were analysed, 93 (20.6\%) were HIV-positive, with 15/93 (16.1\%) being recently infected. Recent infection was independently associated with genital ulcer disease at presentation, especially ulcers with no detectable STI pathogens. Among the 78 (83.9\%) with long-term infection, only 30 (38.5\%) were on ART, with 23/30 (76.7\%) virally suppressed.

Conclusions. In a population at risk of HIV transmission, there was a high burden of recent infection and unsuppressed VLs. Incorporating pre-exposure prophylaxis, ART initiation and adherence support into STI services will be necessary for progress towards eliminating HIV transmission.
\end{abstract}

S Afr Med J 2022;112(2):96-101. https://doi.org/10.7196/SAMJ.2022.v112i2.15930

Sexually transmitted infections (STIs) other than HIV are a global public health problem. In addition to causing complications such as inflammation, scarring and subfertility associated with upper genital tract infections and transmission from mother to child, some STIs are associated with an increased risk of HIV acquisition or transmission. ${ }^{[1]}$ Genital inflammation from both ulcerative and non-ulcerative STIs leads to local recruitment of CD4 cells, macrophages and dendritic cells, which are target cells for HIV. ${ }^{[2]}$ Mucosal disruption associated with ulcerative genital infection facilitates HIV entry, leading to increased susceptibility to HIV acquisition. ${ }^{[2,3]}$ For people living with HIV, engaging in sex without a condom while HIV plasma viral loads (VLs) are $>200$ copies/mL with or without an STI has been associated with transmission of HIV to an uninfected partner. ${ }^{[4-7]}$

South Africa (SA) has a high burden of both HIV and other STIs. ${ }^{[8,9]}$ Although the country had a $49 \%$ decline in new HIV infections between 2010 and 2019, this drop has not been sufficient to meet global HIV prevention targets. ${ }^{[9]}$ Better integration of HIV and STI prevention and treatment services has been proposed to accelerate progress towards the goal of zero new HIV infections.

\section{Objectives}

We describe HIV positivity, antiretroviral therapy (ART) use, viral suppression and recency of HIV infection among symptomatic STI service attendees in SA and discuss the implications of our findings for HIV and STI prevention, care, treatment and surveillance.

\section{Methods}

Setting

This study was nested in the ongoing sentinel site-based aetiological surveillance of STIs conducted by the National Institute for Communicable Diseases (NICD). In a country in which STI care is syndromic, the main objectives of this surveillance are to monitor trends in the aetiological causes of the major STI syndromes, genital ulcer syndrome (GUS), male urethritis syndrome (MUS) and vaginal discharge syndrome (VDS), validate the recommended treatment algorithms and regimens, and ensure that current regimens cover the most common causative STI pathogens for each syndrome. During the period 1 January - 31 December 2019, sentinel surveillance was conducted at two primary healthcare centres located in two provinces 
and aimed to enrol 150 males with MUS, 100 females with VDS and 100 individuals with GUS per site.

\section{Design}

In a cross-sectional study, consecutive male and female STI service attendees aged $\geq 18$ years presenting with genital discharge or ulcers were enrolled at the two sentinel surveillance sites.

\section{Data collection}

During the surveillance period, primary care nurses referred symptomatic STI service attendees to surveillance officers professional nurses placed at the sentinel sites during regular clinic operating hours - for eligibility assessment. Following eligibility assessment and informed consent procedures, eligible males and females were enrolled. Demographic and clinical information was collected using a surveillance officer-administered questionnaire. Demographic variables included age and gender; behavioural variables included condom use at last sexual encounter, having sexual intercourse with a non-regular sexual partner during the preceding 3 months, and having sexual partners living outside the attendee's province or country during the preceding 3 months. Clinical variables were clinical syndrome(s) diagnosed on the day of enrolment, a history of STI syndromes during the preceding 12 months, non-resolution of STI symptoms during the preceding 3 months, referral from another STI treatment provider, knowledge of HIV status, date of most recent HIV status, and self-reported HIV status. Male attendees were asked whether they had been circumcised, and the circumcision method if they had. Circumcision status was confirmed at genital examination. Participants were asked to provide genital specimens - endourethral swabs, cervicovaginal swabs and ulcer swabs for all those with urethral discharge, vaginal discharge and genital ulcers, respectively. All attendees provided two $10 \mathrm{~mL}$ venous blood specimens for laboratory testing. Tests conducted in the HIV and STI reference laboratory included tests for the following biomarkers: screening and confirmatory HIV serology (enzyme immunoassay), Western blot and HIV VL for specimens that were HIV-positive on serology, limiting-antigen avidity (LAg) testing for recency of infection among Western blot-positive specimens, and detection of plasma antiretrovirals (ARVs) on all HIV serologypositive specimens.

\section{Laboratory procedures}

DNA was extracted from the genital swabs and tested using a validated in-house real-time multiplex polymerase chain reaction (PCR) assay on the RotorGene platform (Qiagen, Germany) to detect the presence of the following STI pathogens: Neisseria gonorrhoeae, Chlamydia trachomatis, Trichomonas vaginalis, Mycoplasma genitalium from the MUS and VDS swab specimens, and herpes simplex virus (HSV), Treponema pallidum, Haemophilus ducreyi and L1, L2 and L3 serovars of C. trachomatis from the genital ulcer swab specimens. HSV-1 and 2 subtyping was performed using a commercial PCR assay (Sacace Biotechnologies, Italy). Rapid plasma reagin (RPR) seropositivity was determined using the Immutrep RPR assay (Omega Diagnostics, UK), while HSV-2 seropositivity was determined using the Focus HerpeSelect 2 ELISA IgG assay (Focus Diagnostics, USA).

HIV status was determined using two fourth-generation enzyme immunoassays, Vironostika HIV Uniform II antigen/antibody (Biomerieux, Netherlands) and the Elecsys HIV Combi PT assay (Roche, Germany). Western blot status was determined using a nitrocellulose test strip to which test samples and controls were added; this was incubated for an hour and processed on an automated instrument (Autoblot 3000H; MedTec BioLab Equipment, USA).
Colour development was observed in specific areas on the test strip where the various protein bands were immobilised. The major bands of significance were gp160, gp120, gp41 and $\mathrm{p} 24$. A positive result was reported when at least two major bands were present, i.e. gp160 and/or gp120, gp41 or p24. An indeterminate result was reported when one or more bands were present but the results did not meet the criteria for a positive result. A negative result was reported when no bands were present. Recency of HIV infection was determined using the Maxim HIV-1 Limiting Antigen Avidity assay (Maxim Biomedical, USA). The normalised optical density (ODn) value determined whether a sample was classified as long-term or recent infection. Samples with an ODn $>2.0$ were considered longterm infections. If the ODn was $\leq 2.0$, the samples were re-tested in triplicate, i.e. confirmatory testing. If the ODn of the sample in confirmatory testing was $<1.5$, the sample was considered a recent infection. If the sample ODn was $\geq 1.5$ after confirmatory testing, it was considered a long-term infection. In a study including samples from participants with known dates of seroconversion, the Maxim assay was found to have a false recent rate (FRR) (i.e duration of infection $>6$ months) of $2.17 \%$ at an ODn of $<1.5$ with no VL criteria included, and an FRR of $1.12 \%$ at the same ODn, VL $>1000$ copies/ $\mathrm{mL}$ and no ARVs in plasma. ${ }^{[10]}$ HIV VL was measured using the COBAS AmpliPrep/COBAS TaqMan HIV-1 Test, version 2.0 (CAP/ CTM v2.0) (Roche, Germany). The test can quantify HIV-1 RNA over the range of 20 copies to 10 million copies from a $1.0 \mathrm{~mL}$ ethylenediamine tetraacetic acid (EDTA) plasma input sample. Pooled nucleic acid amplification testing (NAAT) followed by disaggregation to test for individual samples included in a positive pool was used. Pool sizes of 10 samples were used. The pooled testing and the individual NAAT testing was performed using the CAP/CTM v2.0. The dynamic range of the test is $20-10$ million copies $/ \mathrm{mL}$ from $1.0 \mathrm{~mL}$ of pooled plasma (10 individual samples of $0.1 \mathrm{~mL}$ each). The presence of ARV analytes in plasma was detected using a high-performance liquid chromatography with tandem mass spectrometry assay which was developed and validated for screening dolutegravir, efavirenz, lopinavir and nevirapine in human plasma. The assay was developed at the Division of Clinical Pharmacology, University of Cape Town. Sample preparation consisted of a protein precipitation extraction procedure followed by high-performance liquid chromatography with tandem mass spectrometry detection using gradient elution. Dolutegravir-d4, efavirenz-d5, lopinavir-d8 and nevirapine-d 3 were used as internal standards. An API 4000 mass spectrometer (AB Sciex, USA) at unit resolution in the multiple reaction monitoring mode was used to monitor the transition of the protonated precursor ions at mass/charge ratio $(\mathrm{m} / \mathrm{z}) 420.1$, 316.0, 629.5 and 267.1 to the product ions at $\mathrm{m} / \mathrm{z} 277.2,243.9,447.2$ and 226.0 for dolutegravir, efavirenz, lopinavir and nevirapine, respectively. The quality control cut-off concentration for positivity of each analyte was set at $0.02 \mu \mathrm{g} / \mathrm{mL}$ to allow for adequate ARV detection. Two sets of quality control samples were analysed during inter- and intra-validation $(2 \times n=18)$. The percentage difference for all drugs was $<2.6 \%$ between sets and the precision (percent coefficient of variation) was $<11.5 \%$. The assay was validated to detect analytes of dolutegravir, efavirenz, lopinavir and nevirapine because these were the integrase, protease and non-nucleoside reverse transcriptase inhibitor drugs added to the backbone of all public sector first- or second-line regimens during the surveillance period. These ARVs would not be taken for pre-exposure prophylaxis.

\section{Data management and analysis}

Completed questionnaires were sent to the data centre in the STI reference laboratory at the NICD. Data were entered into a study- 
specific Microsoft Access 2016 database (Microsoft, USA). Data were then exported to Stata version 14.2 (StataCorp, USA) for analysis. Descriptive statistics were used to describe enrolled participants, comparing HIV-positive long-term or recently acquired with HIV-negative attendees. A recent infection algorithm taking into account HIV serology, Western blot, LAg assay, HIV VL and absence of ARV analytes in plasma was used to assign HIV-positive individuals as recently infected or not. An individual who: (i) was HIV-positive or negative on confirmatory enzyme immunoassays; (ii) was positive on the Western blot assay; (iii) was classified as LAg assay recent - as determined by an ODn of >1.5; (iv) had a VL >1 000 copies/ $\mathrm{mL}$; and $(v)$ had no ARV analytes detected in plasma was considered recently infected. An HIV-positive individual with a plasma $\mathrm{VL} \leq 200$ copies/ $\mathrm{mL}$ was considered to have a suppressed VL. Univariable and multivariable Poisson regression models with robust error variance were used to determine the demographic, behavioural and clinical factors associated with: (i) being HIVpositive and recently infected compared with being HIV uninfected; (ii) being HIVpositive with long-term infection compared with being HIV uninfected; and (iii) being HIV-positive and recently infected compared with HIV-positive with long-term infection. Variables that had $p$-values $<0.1$ in univariable analyses were included in the multivariable models in a stepwise backward fashion until the equivalent of one variable per 10 outcomes remained in the models. Age was used as a continuous variable and included in all multivariable models a priori.

\section{Results}

Between 1 January and 31 December 2019, a total of 494 symptomatic STI service attendees were enrolled at the two sentinel sites, located in Gauteng and Western Cape provinces, respectively. Of the 494 enrolled, 451 (91.3\%) had adequate plasma specimens for HIV biomarker measurement (Fig. 1). The median (interquartile range (IQR)) age of those tested was $29(25-35)$ years, with $101(22.4 \%)$ aged $\leq 24$ years, $313(69.4 \%)$ male, and 109 (24.2\%) enrolled at the Western Cape site.

Of the 451 attendees tested by enzyme immunoassay in the HIV reference laboratory, 93 (20.6\%) were HIV-positive on serology, while 358 (79.4\%) were HIVnegative. Among the HIV-positives, 88/93 (94.6\%) were Western blot-positive while $5 / 93(5.4 \%)$ were Western blot-negative (Fig. 1). Of the 5 who were Western blot-

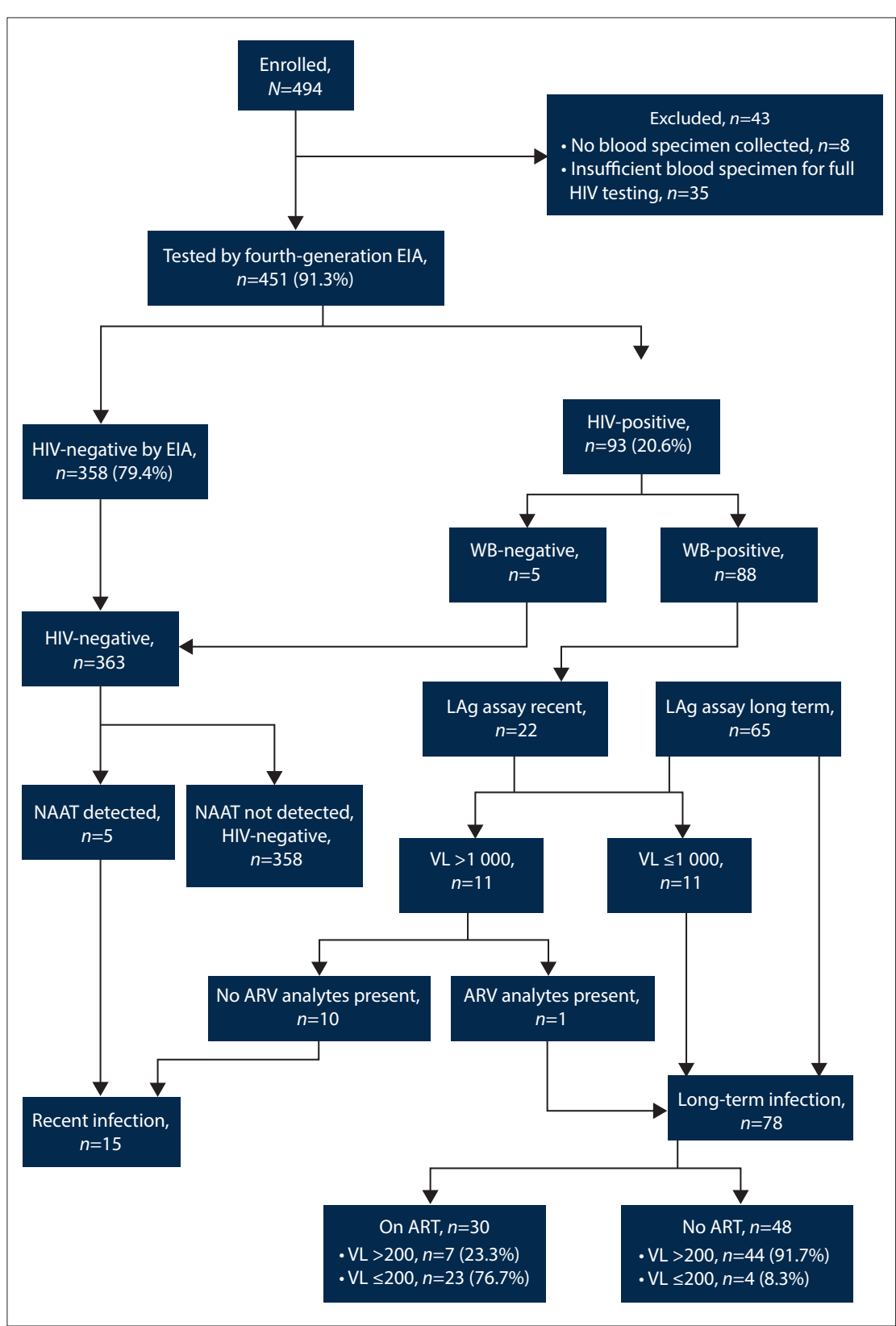

Fig. 1. Study flow. (EIA = enzyme immunoassay; $W B=$ Western blot; LAg = limiting-antigen avidity assay; NAAT = nucleic acid amplification testing; $V L=$ viral load; $A R V=$ antiretroviral; $A R T=$ antiretroviral therapy.)

negative and tested along with all the other HIV-negatives for presence of HIV RNA using nucleic acid amplification, all were NAAT-positive, indicating acute HIV infection. Of the 88 who were Western blotpositive, 22 were classified as having recent HIV infection on the LAg assay, although only 11 went on meet the criteria for recent infection based on VL levels $>1000$ copies/ $\mathrm{mL}$. In total, there were 15 positive individuals (16.1\%) who met the criteria for recent HIV infection. Only 2/15 individuals who met the criteria for recent infection correctly reported being HIV-positive and none were on ART, either by ARV exposure or self-report. The median (IQR) VL among those who were recently infected was $\log _{10} 5.8$ (4.8 - 6.7). Table 1 describes HIV-positive and negative attendees, comparing HIV-positive, recently infected patients with those who were HIV-negative. Compared with being HIV-negative, being recently infected was independently associated with a genital ulcer presentation (adjusted prevalence rate ratio (aPRR) 3.48 (95\% confidence interval (CI) 1.24 - 9.73); $p=0.017$ ) in a model adjusting for age and having genital ulcers at presentation. Being recently infected was also associated with no detectable STI-related ulcer pathogens (aPRR 4.67 (95\% CI 1.18 - 18.42); 


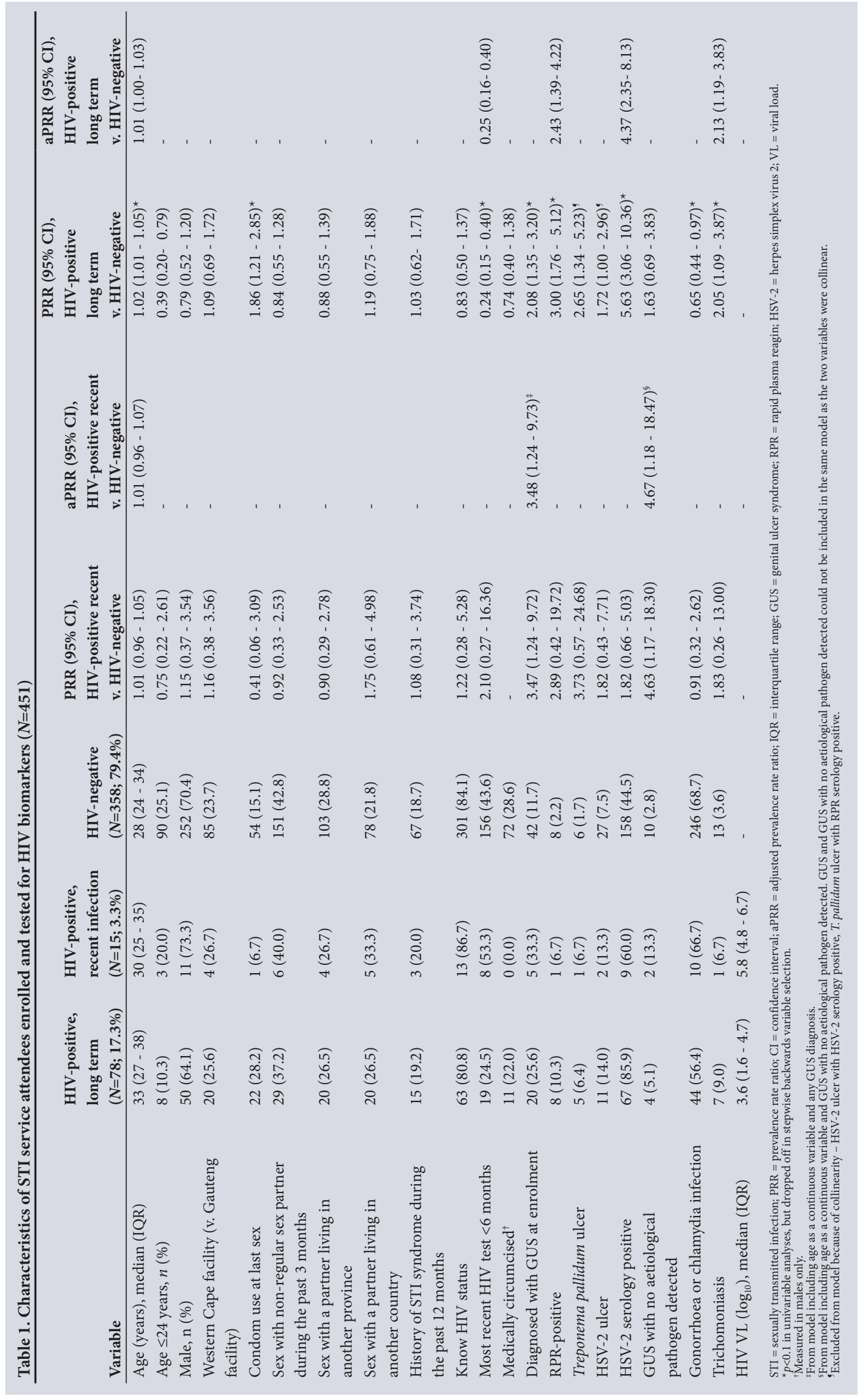


$p=0.024)$ in a model adjusting for age and ulcers with no detectable pathogens. There was a trend towards syphilitic ulcers, RPR positivity and having tested for HIV during the 6 months preceding enrolment being associated with being recently infected in univariable analyses, although the associations did not reach statistical significance. Of the 78 attendees who met the criteria for long-term HIV infection (83.9\% of those who were HIV infected), $30(38.5 \%)$ had plasma ARVs present and were considered to be on ART, while 48 (61.5\%) were not. As expected, the proportion of individuals with suppressed VLs was higher among those who were on ART compared with those who were not $(n=23 / 30$ (76.7\%) v. $n=4 / 48(8.3 \%) ; p<0.001)$. Although viral suppression was higher among those on ART, the level of viral suppression was below the then Joint United Nations Programme on HIV/AIDS (UNAIDS) target of $90 \%$. Of those who had plasma ARVs detected $(n=30)$, $21(70.0 \%)$ self-reported being on ART, indicating under-reporting of HIV status and ART use. Comparing attendees who were HIVpositive with long-term HIV infection $(n=78)$ with those who were HIV-negative $(n=358)$, those who were HIV-positive were less likely to have tested for HIV during the preceding 6 months (aPRR 0.25 (95\% CI $0.16-0.40$ ); $p<0.001$ ), more likely to be RPR serology-positive (aPRR 2.43 (95\% CI 1.39 - 4.22); $p=0.002$ ) and HSV-2 serology-positive (aPRR 4.37 (95\% CI 2.35 - 8.13; $p<0.001$ ), and more likely to have $T$. vaginalis infection on genital discharge specimens (aPRR 2.13 (95\% CI 1.19 - 3.83); $p=0.011$ ) in a model adjusting for these variables as well as age.

Comparing HIV recently infected with long-term infected attendees, individuals who met the criteria for recent infection were more likely to have tested for HIV during the preceding 6 months (aPRR 1.76 (95\% CI $1.10-2.81) ; p=0.018$ ) in a model adjusting for age and HIV testing during the preceding 6 months. There was also a trend towards the recently infected being less likely to be HSV-2seropositive (aPRR 0.38 (95\% CI $0.13-1.11) ; p=0.077$ ) in a model adjusting for age and HSV-2 seropositivity, but this did not reach statistical significance.

\section{Discussion}

This study of symptomatic STI service attendees found HIV positivity of $20 \%$, with close to one-sixth of HIV infections being recent. None of those who met the criteria for recent infection self-reported being on ART. The study also found suboptimal ART coverage among those with long-term HIV infection, and suboptimal viral suppression among those on ART. The median HIV VL was high among those who were HIV-positive (in both long-term and recently infected groups), placing attendees at high risk of transmitting HIV to seronegative partners. Recent infection status was independently associated with genital ulcers, particularly those of unknown aetiology, when compared with HIV-negative status, but this association was not apparent when recent infection status was compared with long-term HIV infection status. We have previously found a high burden of genital ulcers of unknown aetiology in similar surveillance, and acute HIV ulceration could be a cause of these ulcers. ${ }^{[1]}$

The majority of symptomatic STI service attendees were HIVseronegative but at risk of acquiring HIV, as they presented with signs of genital tract inflammation and a significant proportion had active, untreated STIs. These findings strengthen the case for better integration of HIV prevention and care services with STI prevention, care and treatment. Integrated STI services should include HIVrelated services such as counselling and testing, ART initiation, adherence support, VL measurement and HIV drug resistance testing where indicated. Given the high burden of recent HIV infection, which may suggest a high incidence in the catchment population of the sentinel clinics, pre-exposure prophylaxis (PrEP) services should be urgently integrated into STI services. Referrals or provision of on-site voluntary medical male circumcision services should also be expanded. Injectable ARVs being developed and studied for use as PrEP offer a promising intervention for inclusion within similar STI services. ${ }^{[12]}$

\section{Study limitations}

Our study was subject to some limitations. First was the small sample size, which was a result of logistical challenges encountered in setting up the second surveillance site and the large proportion of specimens that were inadequate. This small sample size meant a small number of outcomes and less power to detect some clinically and epidemiologically relevant associations between recent infection and HIV-negative status as well as recent infection and longterm infection status. Second, the study only included two clinics, one in Gauteng and the other in the Western Cape, limiting the generalisability of findings to other clinics in different settings. Lastly, this was a cross-sectional study with no follow-up. This meant that we were unable to determine temporal associations between recency of infection and STIs - for example, the association of recent infection with ulcers with no associated STI-related pathogens detected may be a marker of acute HIV infection and not a risk factor for HIV acquisition.

\section{Conclusions}

Despite the above limitations, our study serves as a reminder of the value of STI services as a platform for identifying newly infected individuals with high VLs for rapid ART initiation, individuals with long-term infection not on ART for treatment initiation and linkage to care, and those with suboptimal viral suppression for adherence counselling and enhanced treatment support. It is important to improve the effectiveness of ART as HIV prevention in those who are already HIV infected by ensuring viral load suppression among all on treatment, while identifying individuals at risk of HIV acquisition for biomedical prevention interventions. Our study also highlights the utility of integrated STI services catering to both HIV-positive and HIV-negative individuals for surveillance of the coverage of key interventions for the biomedical prevention of both HIV and STIs in similar settings.

\section{Declaration. None.}

Acknowledgements. The authors thank Alex Vezi, Valencia Kekana and Bubele Makheleni for assistance with data collection.

Author contributions. TK conceptualised the nested study, FR oversaw data collection in the field, and EC, MG, DG, LW and VM oversaw laboratory procedures for HIV and STI testing, supervised by RK and AP. TK analysed the data and drafted the manuscript. All authors critically reviewed and revised the manuscript and approved it for submission.

Funding. The study was funded by in part by the National Department of Health through a core funding grant to the Centre for HIV and STIs and the Polio Research Foundation through research grant 18/03 awarded to TK. The University of Cape Town Clinical Pharmacokinetic Laboratory is supported in part via the Adult Clinical Trial Group (ACTG), by the National Institute of Allergy and Infectious Diseases (NIAID) of the National Institutes of Health under award numbers UM1 AI068634, UM1 AI068636 and UM1 AI106701, as well as by the Infant Maternal Pediatric Adolescent AIDS Clinical Trials Group (IMPAACT), funding provided by the National Institute of Allergy and Infectious Diseases (U01 AI068632), the Eunice Kennedy Shriver National Institute of Child Health 
and Human Development, and National Institute of Mental Health grant AI068632. The content is solely the responsibility of the authors and does not necessarily represent the official views of the sponsors.

\section{Conflicts of interest. None.}

1. Røttingen J-A, William Cameron D, Garnett GP. A systematic review of the epidemiologic interactions between classic sexually transmitted diseases and HIV: How much really is known? Sexually Transm Dis 2001;28(10):579-597. https://doi.org/10.1097/00007435-200110000-0000

2. Mtshali A, Ngcapu S, Mindel A, Garrett N, Liebenberg L. HIV susceptibility in women: The roles of genital inflammation, sexually transmitted infections and the genital microbiome. J Reprod Immunol 2021;145:103291. https://doi.org/10.1016/j.jri.2021.103291

3. Wall KM, Karita E, Nyombayire J, et al. Genital abnormalities, hormonal contraception, and HIV transmission risk in Rwandan serodifferent couples. I Infect Dis 2021:224(1):81-91, https:/doi. org/10.1093/infdis/jiab071

4. Rodger AJ, Cambiano V, Bruun T, et al. Sexual activity without condoms and risk of HIV transmission Rodger AJ, Cambiano V, Bruun T, et al. Sexual activity without condoms and risk of HIV transmission
in serodifferent couples when the HIV-positive partner is using suppressive antiretroviral therapy. JAMA 2016;316(2):171-181. https://doi.org/10.1001/jama.2016.5148

5. Rodger AJ, Cambiano V, Bruun T, et al. Risk of HIV transmission through condomless sex in serodifferent gay couples with the HIV-positive partner taking suppressive antiretroviral therapy (PARTNER): Final results of a multicentre, prospective, observational study. Lancet 2019;393(10189):2428-2438. https://doi.org/10.1016/s0140-6736(19)30418-0
6. LeMessurier J, Traversy G, Varsaneux O, et al. Risk of sexual transmission of human immunodeficiency virus with antiretroviral therapy, suppressed viral load and condom use: A systematic review. Can Med Assoc J 2018;190(46):E1350-E1360. https://doi.org/10.1503/cmaj.18031

7. Attia S, Egger M, Müller M, Zwahlen M, Low N. Sexual transmission of HIV according to viral load and antiretroviral therapy: Systematic review and meta-analysis. AIDS 2009;23(11):1397-1404. https:// doi.org/10.1097/qad.0b013e32832b7dca

8. Kularatne RS, Niit R, Rowley J, et al. Adult gonorrhea, chlamydia and syphilis prevalence, incidence, treatment and syndromic case reporting in South Africa: Estimates using the Spectrum-STI model, 1990 - 2017. PLOS ONE 2018;13(10):e0205863. https://doi.org/10.1371/journal.pone.0205863

9. Global HIV Prevention Coalition. Implementation of the HIV Prevention 2020 Road Map. Fourth progress report, November 2020. https://www.unaids.org/en/resources/documents/2020/fourthannual-progress-report-of-the-global-hiv-prevention-coalition (accessed 5 August 2021).

10. Sempa JB, Welte A, Busch MP, et al. Performance comparison of the Maxim and Sedia limiting antigen avidity assays for HIV incidence surveillance. PLoS ONE 2019;14(7):e0220345. https://doi. org/10.1371/journal.pone. 0220345

11. Kularatne RS, Muller EE, Maseko DV, Kufa-Chakezha T, Lewis DA. Trends in the relative prevalence of genital ulcer disease pathogens and association with HIV infection in Johannesburg, South Africa, 2007 - 2015. PLoS ONE 2018;13(4):e0194125. https://doi.org/10.1371/journal.pone.0194125

12. Clement ME, Kofron R, Landovitz RJ. Long-acting injectable cabotegravir for the prevention of HIV infection. Curr Opin HIV AIDS 2020;15(1):19-26. https://doi.org/10.1097/coh.0000000000000597

Accepted 12 October 2021 